\title{
Investigation of energy efficient protocols based on stable clustering for enhancing lifetime in heterogeneous WSNs
}

\author{
Noor Alhuda F. Abbas', Jaber H. Majeed ${ }^{2}$, Waleed Khalid Al-Azzawi ${ }^{3}$, Adnan Hussein Ali ${ }^{4}$ \\ ${ }^{1}$ Department of Computer Techniques Engineering, Al-Esraa university college, Baghdad, Iraq \\ ${ }^{2}$ Department of Electrical Engineering, University of Technology, Baghdad, Iraq \\ ${ }^{3}$ Department of Medical instruments engineering techniques, Al-Farahidi University, Baghdad, Iraq \\ ${ }^{4}$ Middle Technical University, Institute of Technology, Baghdad, Iraq
}

\begin{tabular}{|c|c|}
\hline Article Info & ABSTRACT \\
\hline Article history: & There are certain challenges faced with wireless sensor networks (WSNs) \\
\hline Received Apr 4, 2021 & $\begin{array}{l}\text { performances, consumption can be seen amongst all these challenges as a } \\
\text { serious area of research. Data from sensor nodes are transmitted by most }\end{array}$ \\
\hline Revised Jul 13, 2021 & WSN energy either among many nodes or to the base station (BS), and due \\
\hline Accepted Aug 27, 2021 & $\begin{array}{l}\text { this connection, several routing protocols were developed for supporting in } \\
\text { data transmission in the WSNs. Extending network lifetime in an operational }\end{array}$ \\
\hline Keywords: & or exchanging sensor node batteries is almost impossible. Energy balancing \\
\hline CCS \& SEP & and energy efficiency are significant research scopes as per designing of \\
\hline Energy efficient protocol & one where every node has different amount of energy linked to it before it is \\
\hline Heterogeneous WSN & deployed in a network. Therefore, different energy efficient routing protocols \\
\hline HPEEA protocol & $\begin{array}{l}\text { have been proposed which enables lesser consumption of energy, longer } \\
\text { stability period which leads to the network lifetime increasing. In this study, } \\
\text { the average energy of a WSN is computed after every logical round of } \\
\text { operation for our protocol-HPEEA and compare it with two well-known } \\
\text { heterogeneous protocols namely-SEP and CCS. At the end of the considered } \\
\text { number of logical operations, MATLAB with simulation results confirm that } \\
\text { HPEEA protocol have a reduction in the energy consumption compared to } \\
\text { other protocols. }\end{array}$ \\
\hline
\end{tabular}

This is an open access article under the CC BY-SA license.

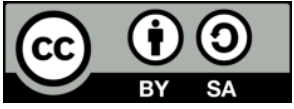

\section{Corresponding Author:}

Adnan Hussein Ali

Middle Technical University

Institute of Technology-Baghdad, Iraq

Email: dradnanmtu@gmail.com

\section{INTRODUCTION}

Recently, many researchers are attracted to the area of wireless sensor networks (WSNs) because of their participation in different practical applications [1]. There are a number of features that WSNs possess Which enables them different from further normal networks. For instance, they have limited source energy, computing capacities, and the data management [2]. Therefore, during use, the features of WSNs must be considered. To send collected data of WSN to the base station (BS), several protocols have been used for routing had introduced and this, in turn, sends it to the end-user [3].

WSNs can be considered as a developing paradigm of ad-hoc wireless networks. Such networks are made up of hundreds up to thousands of independent equipment, known as sensor node. Generally, each sensor node has a low-power restrained device having a processor with inadequate power to process, a memory of restricted amount, a battery that supplying power, an RF wireless communication, and a sensor 
board which is made up of various kinds of sensing. Generally, batteries are used to power sensor nodes if organized are abandoned with significant time period and usually its functions for months to years [4]. Hence, energy is not a sufficient resource in WSN and therefore to extend the lifetime of the entire sensor, effective usage is critical. Recently, the comprehensive using of WSNs, which are self-organized have been increasing due to improve performances of the microminiature sensor from were memory capability and sensitivity, also a decreasing in prices [5]. They are made up of many sensors, controlling the area of interest and to get the information exchange, special routing protocols are used. Using aircraft and organize networks, the sensors are usually deployed randomly in tough environments by themselves. Because of the advantages of WSNs which include; low price, self-organization and convenient deployment, a detection of forest fire [6], in the environment controlling and monitoring [7], in the field of healthcare and medical systems [8], and smart homes [9].

Recent studies in WSNs intends to overcome these limitations by creating new or improving existing protocols, introduction of new design concepts, developing new algorithms and building new applications. To accomplish these WSN requirements, schemes are being developed by researchers presently. Energy consumption is one of the biggest problems of WSN's. This needs to be carefully investigated and researched for its consumption patterns at each respective sensor node of the network to improve and build appropriate algorithms for optimum consumption of energy and to maximize the network lifetime [10].

The sensors batteries are impossible for changing by peoples, due to severe environment of the positioned sensors at some implementations. Thus, in order to design routing protocols, energy efficiency is an important factor. Many studies have focused on energy conservation and have proposed a lot of routing schemas. The sensors are divided into two types, by two-tier routing schema: member nodes in additions to cluster heads $\mathrm{CHs}$ [11]. With this two-tier WSN, the sensor data have been sent to the corresponding $\mathrm{CH}$ from member nodes with single or multiple hop communication, at that moment $\mathrm{CHs}$ will process the data fusion then send the data to the sink. clustering has advantages including [12]:

- Data fusion may be done in CHs in order to fuse the data from their participants, unnecessary data could then be extracted and the transmitting burden of $\mathrm{CHs}$ is eased.

- $\quad$ The network topology is optimized and the controlling messages are minimized so only the related $\mathrm{CH}$ needs to be known by local nodes. Additionally, the networks scalability is increased.

- The topology of the network is simplified and the control messages are reduced because local nodes only need to know its corresponding cluster head. Meanwhile, the scalability of the network is improved.

- The communication bandwidth is retained since a time division multiple access (TDMA) scheme is normally used for intracluster communication.

Sensor nodes are typically provided with a small batteries power which can store restricted energy that can be provided for a short-term time period. The sensor nodes can be generally deployed in remote areas usually left unattended. In such situation, it is difficult or impossible to externally feed or replenish energy to the battery [13]. Thus, an important goal of optimization in this aspect is for the network lifetime to be prolonged with a given energy by the first battery supply along with the network. Therefore, consumption of energy of the whole network in every aspect can be reduced. The possible answer to reduce energy consumption may lie in power aware network, might be the possible answer to reduce energy consumption and it is designed in such a way that each layer of the system can be used strategically [14].

WSNs are seen as an ad-hoc networks form, so that the nodes are mainly sensors besides data sensing have ability to communicate. When deployed, these sensors are equipped with limited power, can work on their own and really do not require any supervision. As a result, the network lifetime is one of the most significant design factors. Clustering can be considered the main energy-efficient methods utilized for increasing the sensor lifetime of a sensor network by reducing sensor nodes consumption of energy [15]. WSN is considered Heterogeneous because it is made up of sensor nodes that had different ability, like various sensing range with computing power. Compared to homogeneous WSN, topology and deployment control are more complicated in heterogeneous WSN.

\section{MATERIAL AND METHODS METHODOLOGY}

\subsection{Wireless sensor network and components of sensor node}

A WSNs are made up of a many of sensors, these sensor nodes are randomly or carefully installed over a geographical zone then networked by wireless links for making a WSN. Ambient condition in the surrounding environment could be measured by sensor nodes and could be managed to mention the characteristics of the phenomena happening at the location that implementing the sensor nodes. Each WSN's sensor nodes have the ability for communicating with each other as well as with the BS aiming for disseminating and integrating data [16]. 
Mainly, WSNs are used in industrial, civilian, in addition to military applications thats concentrated in the field of imaging, battlefield surveillance, target field and intrusion detection. Although, WSNs are currently applied with many civilian application ranges involving home automation, environment and habitant monitoring, traffic control and health applications [17]. WSN is typically consisting of many sensor nodes, that permitting the sensing with higher geographical zones with higher accuracy. Each node is consisting of three key components which are sensing unit, processing unit, in addition to transmission unit as presented in Figure 1 [18].

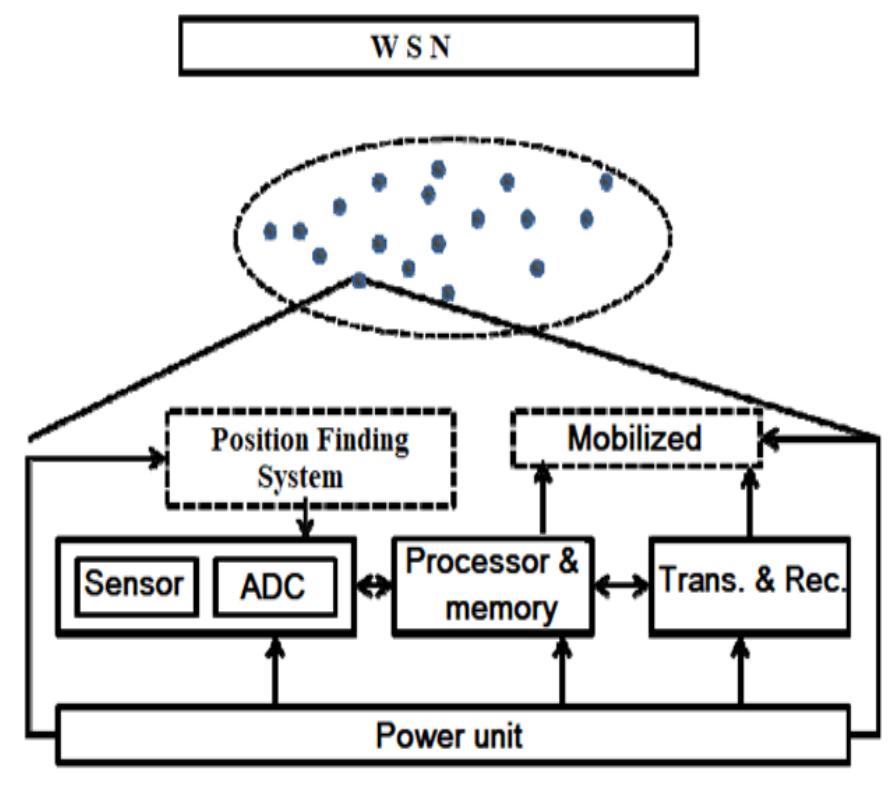

Figure 1. Sensor nodes components at WSN

Methods of increasing energy efficiency are classified into three groups depending on geographical area, which is called coverage method, Green method, concerned with preservation of the environment and exploitation of natural resources and clustering method is the largest group [19], which included the data Centric, hierarchical, and balancing. Section four will discuss theses classification in details. With Regards to clustering, naturally, to satisfy and achieve long lifetime in comprehensive WSN environments and high efficiency of energy, grouping sensor nodes into clusters is widely used by the research community [20].

Each cluster has a leader in hierarchical network structure named cluster head $(\mathrm{CH})$ that carries out the special duties, and many common sensor nodes by means of members [21]. Transferring data in small network is direct and fast, but when the network become large, the sensed data will require many links to get to its destination. And will mostly make conjunctions in the network. Therefore, to make the communication easier and more effective, the network is divided into two clusters or more. An important dilemma in wireless sensor networks is formatting clusters and electing a cluster head and it has a serious effect on energy dissipation in the network [22].

\subsection{Proposed energy efficient protocol for clustering in heterogeneous WSNs}

The protocol-HPEEA focuses on routing in concentric clustering network topology where sensor nodes are classified in concentric clusters. An advanced node and a cluster head, leads each cluster and the performs dissemination and aggregation of data. It is different from EBK protocol and the SEP protocol because it creates concentric clusters based on where the advanced node is located and selects cluster head by K-Theorem method [23].

Concentric clusters are created dynamically by HPEEA routing protocol created on the advanced nodes position in the network, which helps in maximizing the network lifetime. In heterogeneous wireless sensor networks (HWSN), the selection of cluster head based on distance and residual energy, the load distribution amid all the nodes that distributed in the network energy efficiency-oriented routing protocols focuses mainly on achieving maximized networks lifetime. Since energy considers a main constraint in the network, efficient use of limited energy leads to a longer network lifetime [24]. 
Clustering seems one of the conserve energy way of the nodes. Nodes are grouped into clusters in LEACH and are led by a cluster head. Most of the available energy efficiency-oriented routing protocol centered on improving the probabilistic equation for the cluster head selection so as to induce energy parameters in it rather than to plainly depend on heuristic probability. The main aim of the protocol in SEP is to increase the networks stability time, in other words, to delay the period after which the first node in the network dies [25]. The SEP considers as a breakthrough protocol, because in terms of stability it is better than others. Nevertheless, it was found that the time before the death of the networks first sensor node and the time of the networks last dead node. which is the instability time being very low. The gap between the stability and energy efficient protocols in a HWSN can be bridged by this protocol [26]. This can be achieved when both the stability and instability period of the network is increased.

\section{OVERALL METHODOLOGY}

The initialization process must be given before start to explain the implementation of the HPEEA protocol, which is made up of two phases; a first phase is the building phase which three protocols in different algorithms are built in this stage, and the second is the network connection phase which helps in generating a link connection between nodes. Figure 2 presents the total methodology of the paper.

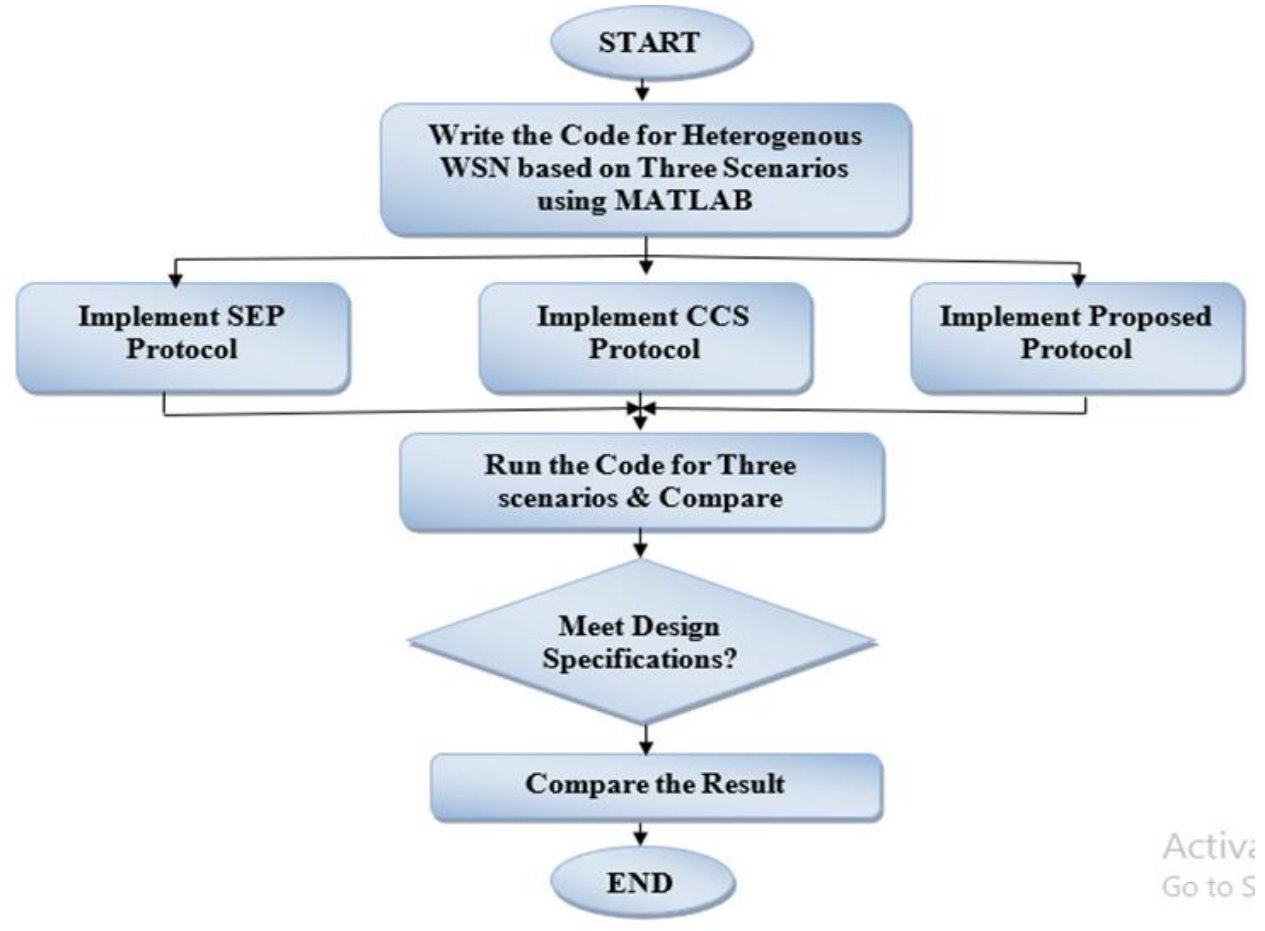

Figure 2. Overall methodology of the protocol HPEEA

\section{IMPLEMENTATION AND PERFORMANCE ANALYSIS}

\subsection{Proposed protocols with varying sensor nodes}

The MATLAB 7.11 simulation tool was applied for simulation implementing the of the proposed protocol (HPEEA). The area of simulation of the field measured is $200 x 100 \mathrm{~m}$. The network is a static tightly organized, since many sensor nodes have been sent out in a two-dimensional topographical space, making a static deployed network. Initially, the energy range of the sensor nodes ranges from 1 to 2 joules and the first energy range of Advanced nodes is $\geq 5$ joules. In different simulation environments, sensor nodes number varies between 100 and 250 so that sensor network performance in these environments is assessed. At every round, the network parameter of dead nodes is calculated and compared with the renowned protocols. In terms of sensor nodes, the varying environments are executed for 5000 rounds.

The simulation results comparing round number against each time the $\mathrm{CH}$ is selected is presented in Figure 3. The simulation is conducted for 5000 numbers of rounds. First simulation is carried out with sensor 
network deploying 100 sensor nodes. The proposed protocol (HPEEA) is compared with the two protocols of SEP and CCS.

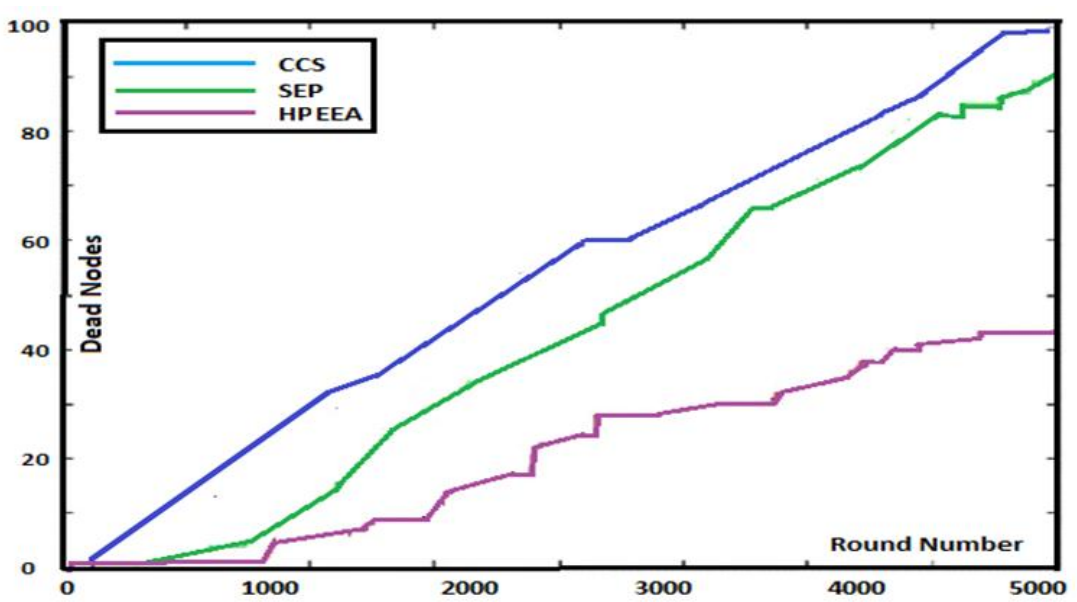

Figure 3. Protocol comparisons for dead nodes number at 100 nodes

In the proposed protocol, the energy dissipation in the nodes is quite low compared to the other two protocols (SEP and CCS). There is a linear increasing in the dead nodes number of SEP and CSS, meanwhile after 4700 rounds in our protocol, the change in the dead nodes is almost negligible. As the rounds number increases, results improve. As the rounds increases, it can be seen that a dead nodes number within our protocol decreases while in the other two protocols the dead nodes rapidly increase.

Figure 3 shows the dead nodes number as the rounds increases. The dead node numbers in SEP, CCS and the proposed protocol (HPEEA) are 95, 86 and 40 respectively at the end of 5000 rounds. According to the above results it can be concluded that (HPEEA) is 55\% more efficient than SEP and 58\% more efficient than CCS.

Figure 4 reveals the simulation results comparing round number against each time the $\mathrm{CH}$ is selected from the available candidate $\mathrm{CH}$. The simulation is conducted for 5000 round numbers. Second simulation is conducted with sensor network having 200 sensor nodes which are randomly arranged. A comparison of the proposed protocol is simulated with two protocols of SEP and CCS. According to the simulation results, a dead node numbers significantly increases as the network nodes number increases in SEP and CCS whereas in our protocol, the increase in the dead node numbers can be neglected.

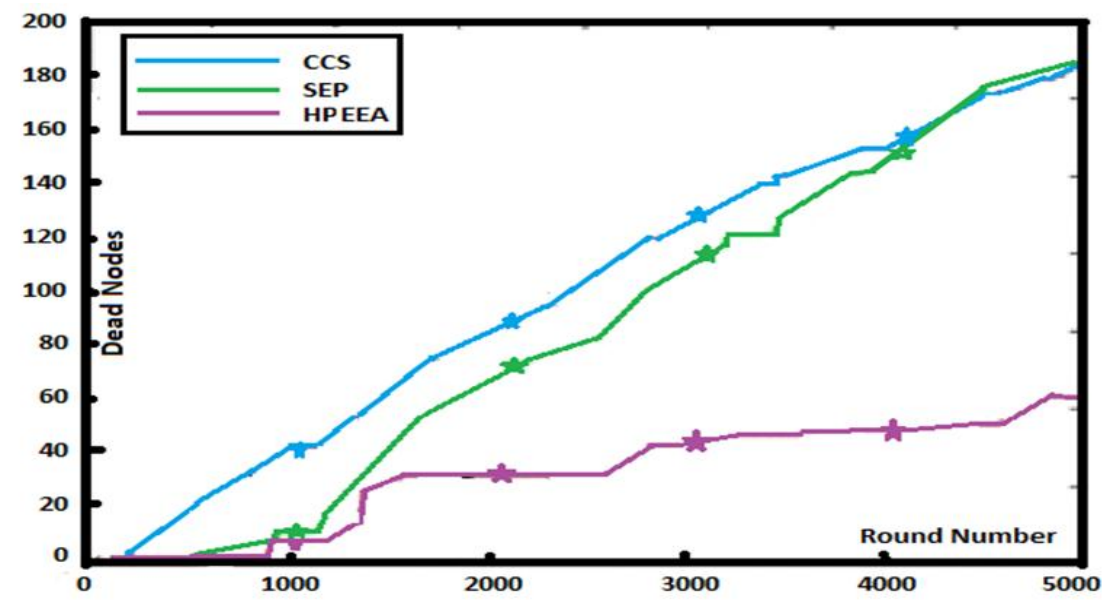

Figure 4. Protocol comparisons for dead node numbers for 200 nodes 
Figure 4 shows the increasing of the round number and its corresponding dead nodes number. The number of dead nodes in SEP, CCS and our protocol are 180, 178 and 45 respectively at the end of 5000 rounds. It can be concluded from the above results that our protocol is $70 \%$ more efficient than SEP and $65 \%$ more efficient than CCS.

Figure 5 reveals simulation results comparing round number against each time the $\mathrm{CH}$ is selected from the available candidate $\mathrm{CH}$. The simulation is conducted for 5000 round numbers. Second simulation is carried out with the sensor network having 300 sensor nodes. These sensor nodes are randomly arranged. A two protocols of SEP and CCS are compared with the HPEEA protocol. The simulation results reveal that dead node numbers significantly increasing as the network node numbers increasing in SEP and CCS while an increasing in the dead node numbers can be neglected in HPEEA protocol. The dead node numbers in SEP, CCS and proposed protocol are 215, 210 and 82 respectively at the end of 5000 rounds. It can be concluded that our protocol is $61.8 \%$ more efficient than SEP and $60.95 \%$ more efficient than CCS from the results above.

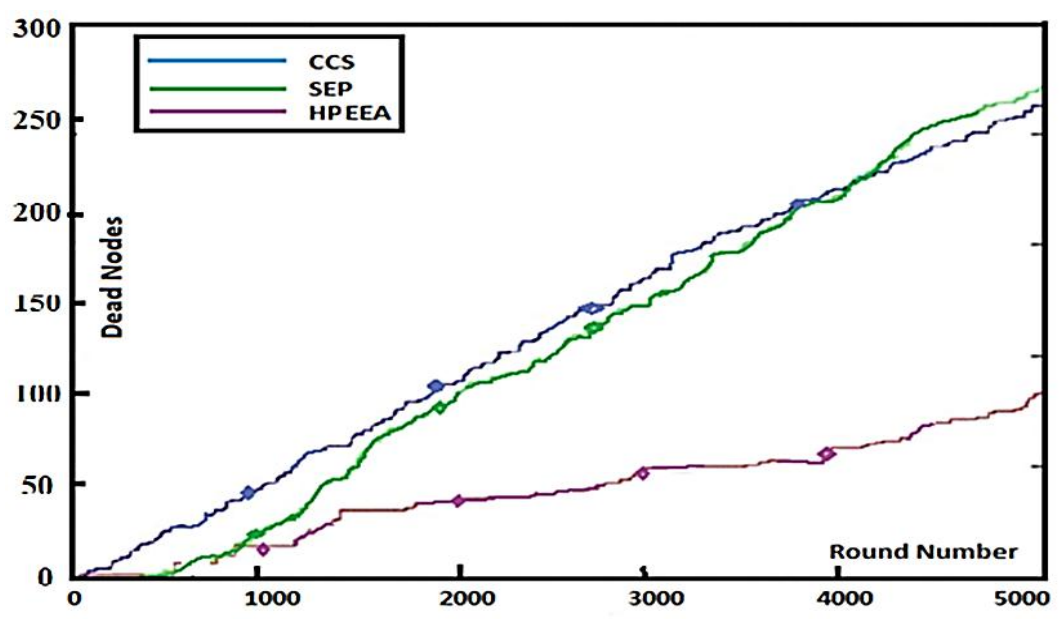

Figure 5. Protocol comparisons for dead node numbers for 300 nodes

From the graphs above, it is seen that as the number of nodes in the network increases, the number of dead nodes at the end of 5000 nodes lower compared to the other protocols in heterogeneous WSN. From the graphs, it is also observed that that although the stability cannot be gotten to that range of SEP, the reason why our protocol is less stable than SEP even though both have two hop routing in protocols to the base station, in SEP, the distance covered to reach the next immediate node collectively is shorter compared to a more static $\mathrm{CH}$ and advanced node scenario in (HPEEA) and also in SEP, $\mathrm{CH}$ changes faster, allowing the nodes to consume lesser energy at closer intervals. Meanwhile, in our protocol the stability period is much better than the CCS protocol. Although, it has discovered that the dead node numbers are much smaller or the lifetime network has been seen to be higher than a SEP or CCS.

\subsection{Combined protocol for energy efficient clustering to enhance WSN lifetime}

The Proposed protocol has to do with design for energy efficient routing protocol in a network such that there is maximization of lifetime by applying duty-cycle. By reducing the dissipation of energy at each round of operation when it is applied with duty-cycle, energy efficiency is achieved. A random number between 0 and 1 is generated by the sensor nodes deployed in the network region, and by comparing with the threshold value, they can be configured. Data aggregation and dissemination to the base station is performed by the cluster head by rotating the role of cluster head throughout the cluster based on its residual energy in addition to the distance for advanced node, the load is balanced among all the sensor nodes within the cluster. An information route of the sensor nodes to the cluster head by using the distance vector routing protocol; which puts together the data as well as its sending to sink node. Robustness regarding node failure are achieved by establishing multi paths to the cluster head from any sensor nodes.

The simulation is done in the area of $500 \mathrm{mx} 500 \mathrm{~m}$. It reveals the results for varying sensor node numbers in the network for lifetime parameter. The simulation results reveal the performance of the proposed protocol when duty cycle applies to the nodes and compared the same without applying duty cycle. The 
network lifetime and energy efficiency is measured as shown in the figures below. Figure 6 reveals the results of the lifetime of the network, simulated for 100,200 and 300 nodes.

Figure 6 , the lifetime of the network with duty cycle is ended at 82 with 100 nodes, and without duty cycle it ends at 31. Similarly, for 200 and 300 nodes, the lifetime ends at 61, 18 and 70, 28 respectively. It can be concluded from the above simulation, that the lifetime of the network is $35.8 \%$ more efficient when nodes are applied with duty-cycle.

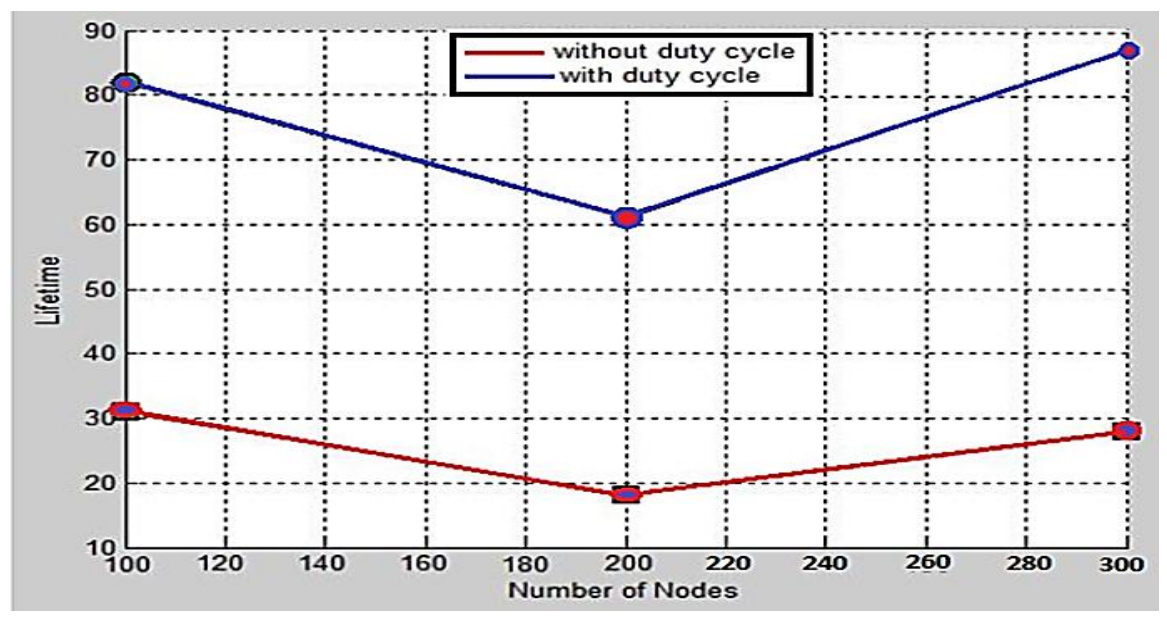

Figure 6. Lifetime comparisons for 100, 200, 300 nodes

Figure 7 shows the lifetime results of the network, simulated for 250,300 and 350 nodes. The graph below shows the network lifetime with duty cycle it ended at 89 with 250 nodes, and without duty cycle it is ended at 19. Similarly, for 300 and 350 nodes, the lifetime ends at 82, 41 and 94, 38 respectively. It can be concluded from the above results that the lifetime of the network is $30.8 \%$ more efficient when nodes are applied with duty-cycle.

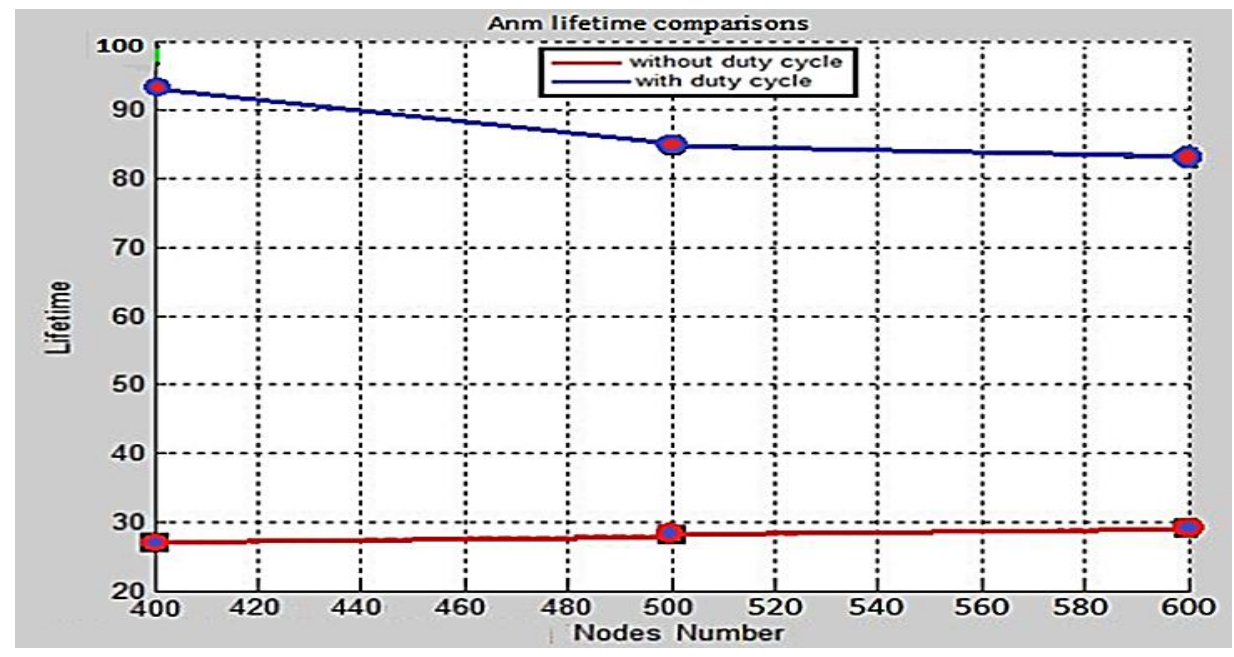

Figure 7. Lifetime comparisons for 600 nodes

Figure 8 presents the final results of the lifetime of the network when nodes of range between 100 500 nodes are deployed in the network. In conclusion, when nodes are applied to the duty cycle, the lifetime of the network is $30 \%$ more efficient. 


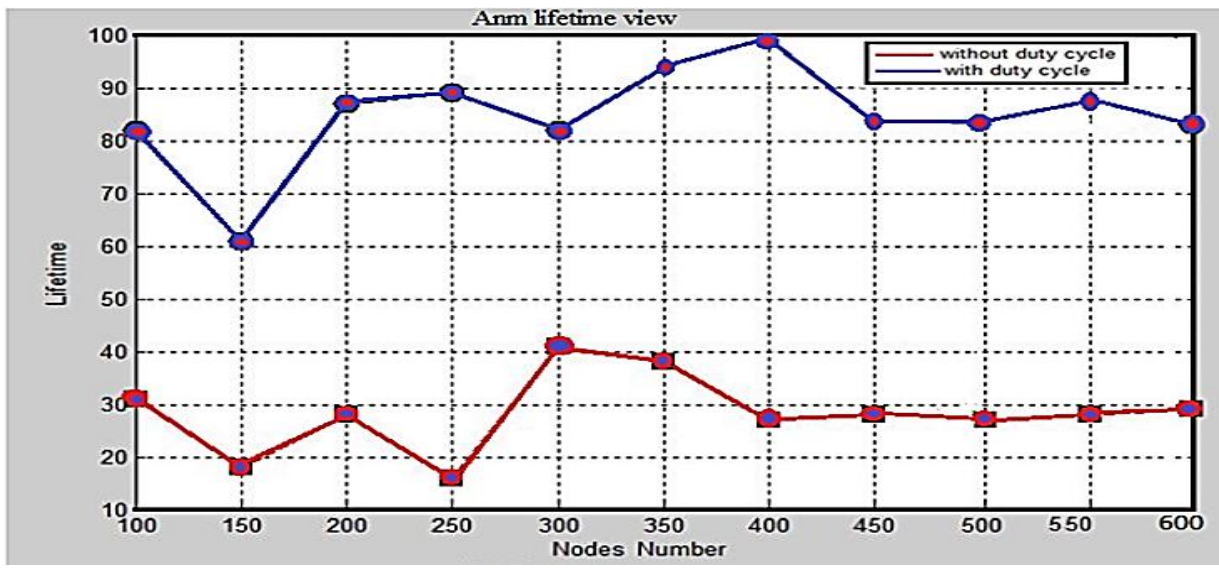

Figure 8. Lifetime comparisons for varying nodes

Combined algorithm by applying duty-cycle, gives $32 \%$ of the average increase in lifetime of network and $34 \%$ of the average increase in the efficiency of energy is observed from the simulation results. From the simulation it can be seen that a network lifetime of the is reduced and efficiency of energy decreases as the node numbers in the network increases, and also if the duty cycle is not applied to the nodes, Therefore, it can be concluded that nodes applying duty-cycle in the combined algorithm can survive for a longer time compared to nodes without applying duty-cycle in this algorithm.

\section{CONCLUSION}

In this paper, a new protocol-HPEEA-technique for data routing over WSNs was proposed and analyzed. The work presented in this research is to provide energy efficient solutions for the design of routing protocols. Routing and clustering protocols investigated claims to improve sensor network lifetime. For efficient operation of wireless networks, Provision of energy methods is an important feature. In this paper, for heterogeneous WSNs, routing protocols are implemented based on clustering protocols for cluster head selected. The proposed protocol for heterogeneous wireless sensor network-HPEEA is created on location information and the sensor nodes residual energy. HPEEA is a scheme which improves the efficiency of energy in heterogeneous networks. The gap between stability and energy efficiency in heterogeneous WSN is narrowed by the proposed HPEEA protocol and its death delays of the first node in the network, therefore increasing the stability period in the network lifetime using stable concentric clustering by choosing the cluster head based on combined rating and the position of the advanced nodes, leveraging K-theorem. Also, with this protocol, as the node numbers in the network increases, the of dead node numbers at the end of 5000 rounds is far less compared to other protocols in heterogeneous WSN. The results show that these two new methods have a long network lifetime comparing with other protocols. Periodic cluster heads selection among all the network nodes or between members of associated cluster, is the main problem of clustering in WSN.

\section{REFERENCES}

[1] C. Li, H. Zhang, B. Hao, and J. Li, "A Survey on routing protocols for large-scale wireless sensor networks," Sensors, vol. 11, no. 4, pp: 3498-2536, 2011, doi: 10.3390/s110403498

[2] I. Zaatouri, A. B. Guiloufi, N. Alyaoui, and A. Kachouri, "A Comparative Study of the Energy Efficient Clustering Protocols in Heterogeneous and Homogeneous Wireless Sensor Networks," Wirel. Pers. Commun., vol. 97, no. 4, pp. 6453-6468, 2017, doi: 10.1007/s11277-017-4847-2.

[3] S. A. Nikolidakis, D. Kandris, D. D. Vergados, and C. Douligeris, "Energy efficient routing in wireless sensor networks through balanced clustering," Algorithms, vol. 6, no. 1, pp: 29-42, 2013, doi: 10.3390/a6010029.

[4] M. M. N. Aldeer, "A summary survey on recent applications of wireless sensor networks," 2013 IEEE Student Conference on Research and Developement, 2013, pp. 485-490, doi: 10.1109/SCOReD.2013.7002637.

[5] G. Abdul-Salaam, A. H. Abdullah, M. H. Anisi, A. Gani, and A. Alelaiwi, "A comparative analysis of energy conservation approaches in hybrid wireless sensor networks data collection protocols," Telecommunication Systems: Modelling, Analysis, Design and Management, vol. 61, no. 12, pp: 159-179, 2016. doi: 10.1007/s11235015-0092-8. 
[6] E. Alnawafa and I. Marghescu, "New energy efficient multi-hop routing techniques for wireless sensor networks: Static and dynamic techniques," Sensors, vol. 18, no. 6, p. 1863, 2018, doi: 10.3390/s18061863.

[7] M. K. Naji, A. D. Farhood, A. H. Ali, "Novel design and analysis of RF MEMS shunt capacitive switch for radar and satellite communications," Indonesian Journal of Electrical Engineering and Computer Science (IJEECS), vol. 15, no. 2, pp. 971-978, 2019., doi: 0.11591/ijeecs.v15.i2.pp971-978.

[8] S. Rani, J. Malhotra, and R. Talwar, "Energy efficient chain based cooperative routing protocol for WSN," Appl. Soft Comput. J., vol. 35, pp: 386-397, 2015, doi: https://doi.org/10.1016/j.asoc.2015.06.034.

[9] S. K. Mitra, J. Banerjee, P. Ghosh, and M. K. Naskar, "Analysis of network lifetime for wireless sensor network," Int. J. Comput. Appl., vol. 32, no. 1, 2011.

[10] V. Saranya, S. Shankar, and G. R. Kanagachidambaresan, "Energy efficient clustering scheme (EECS) for wireless sensor network with mobile sink," Wirel. Pers. Commun., vol. 100, no. 6, pp. 1553-1567, 2018, doi: 10.1007/s11277-018-5653-1.

[11] Mohannad J. Mnati, Raad F. Chisab, Azhar M. Al-Rawi, Adnan H. Ali, Alex Van den Bossche, “An open-source non-contact thermometer using low-cost electronic components," HardwareX, vol. 9, p. e00183, 2021, doi: doi.org/10.1016/j.ohx.2021.e00183.

[12] D. Wajgi, Nileshsingh, N. V. Thakur, "Load balancing based approach to improve lifetime of wireless sensor network," Int. J. Wirel. Mob. Networks, vol. 4, no. 4, p. 155, 2012.

[13] Z. Manap, B. M. Ali, C. K. Ng, N. K. Noordin, and A. Sali, "A review on hierarchical routing protocols for wireless sensor networks," Wireless Personal Communications, vol. 72, no. 2, pp: 1077-1104, 2013.

[14] K. Musale and S. Borde, "Analysis of Cluster Based Routing Protocol for Mobile Wireless Sensor Network," Int. J. Adv. Trends Comput. Sci. Eng., vol. 2, no. 1, pp. 124-129, 2013.

[15] D. Kumar, T. C. Aseri, and R. B. Patel, "EEHC: Energy efficient heterogeneous clustered scheme for wireless sensor networks," Comput. Communications, vol. 32, no. 4, pp: 662-667, 2009, doi: https://doi.org/10.1016/j.comcom.2008.11.025

[16] B. Elbhiri, R. Saadane, and D. Aboutajdine, "Stochastic and Equitable Distributed Energy-Efficient Clustering (SEDEEC) for heterogeneous wireless sensor networks," Int. J. Ad Hoc Ubiquitous Comput., vol. 7, no. 1, pp. 411, 2011, doi: https://doi.org/10.1504/IJAHUC.2011.037849.

[17] M. A. Saad, Mustafa S. T., M. H. Ali, M. M. Hashim, M. Bin Ismail, and Adnan H. Ali, "Spectrum sensing and energy detection in cognitive networks," Indonesian Journal of Electrical Engineering and Computer Science (IJEECS), vol. 17, no. 1, pp. 465-472, 2020., doi: 10.11591/ijeecs.v17.i1.pp465-472.

[18] F. Bajaber and I. Awan, "Energy efficient clustering protocol to enhance lifetime of wireless sensor network," $J$. Ambient Intell. Humaniz. Comput., vol. 1, no. 4, pp. 239-248, 2010, doi: 10.1007/s12652-010-0019-x.

[19] G. Smaragdakis, I. Matta, and A. Bestavros, "SEP: A Stable Election Protocol for clustered heterogeneous wireless sensor networks," 2nd Int. Work. Sens. Actor Netw. Protoc. Appl., pp:1-11, 2004.

[20] B. Elbhiri, R. Saadane, S. El fldhi and D. Aboutajdine, "Developed Distributed Energy-Efficient Clustering (DDEEC) for heterogeneous wireless sensor networks," 2010 th International Symposium On I/V Communications and Mobile Network, 2010, pp. 1-4, doi: 10.1109/ISVC.2010.5656252.

[21] Jaber H. Majeed, Qais Aish, "A Remote Patient Monitoring based on WBAN Implementation with IOT and Cloud Server," Bulletin of Electrical Engineering and Informatics (BEEI), vol. 10, no. 3, pp: 1640-1647, June 2021, doi: https://doi.org/10.11591/eei.v10i3.1813.

[22] Prashant Krishan 2013, “A Study on Dynamic and Static Clustering Based Routing Schemes for Wireless Sensor Networks," International Journal of Modern Engineering Research, vol. 3, no. 2, pp.1100-1104, 2013.

[23] S. A. Lafta, A. H. Ali, M. M. Kareem, Y. A. Hussein, A. H. Ali, "Performance simulation of broadband multimedia wireless networks simulation based on OPNET," Indonesian Journal Electrical Engineering and Computer Science (IJEECS), vol. 17, no. 1, pp. 1-9, 2020, doi: 10.11591/ijeecs.v17.i2.pp1-9.

[24] S. Arjunan, P. Sujatha, "Lifetime maximization of wireless sensor network using fuzzy based unequal clustering and ACO based routing hybrid protocol," Appl.Intell., vol. 48, pp: 2229-2246, 2018, doi: 10.1007/s10489-0171077-y.

[25] M. M. Kareem, M. Ismail, M. A. Altahrawi, N. Arsad, M. F. Mansor and A. H. Ali, "Grid Based Clustering Technique in Wireless Sensor Network using Hierarchical Routing Protocol," 2018 IEEE 4th International Symposium on Telecommunication Technologies (ISTT), 2018, pp. 1-5, doi: 10.1109/ISTT.2018.8701720.

[26] Anas A. Hussien, Adnan H. Ali, "Comprehensive investigation of coherent optical OFDM-RoF employing 16QAM external modulation for long-haul optical communication system," International Journal Electrical and Computer Engineering (IJECE), vol. 10, no. 3, pp. 2607-2616, 2020, doi: 10.11591/ijece.v10i3.pp2607-2616. 